\title{
Caracterização dos acidentes de trabalho durante a atenção à saúde na região nordeste
}

\author{
Characterization of occupational accidents during health care in \\ the northeast region
}

\author{
Milene Pereira de Souza Santos ${ }^{1}$, Ítalo Ricardo Santos Aleluia ${ }^{2}$
}

\begin{abstract}
RESUMO
Objetivo: Caracterizar a evolução quantitativa dos acidentes de trabalho em atividades de atenção à saúde humana na região Nordeste.

Materiais e Métodos: Trata-se de um estudo ecológico de base territorial geográfica, através de dados secundários extraídos da base de dados do Ministério da Previdência e Assistência Social, no período de 2007 a 2010.

Resultados: Verificou-se que no período estudado, a Bahia apresentou o maior número de acidentes de trabalho registrados. Acidentes típicos com Comunicação de Acidente de Trabalho (CAT) se configuram como os principais no ranking dos motivos/situação em que, dentre as consequências, houve maior predomínio das incapacidades permanentes.

Conclusão: Destaca-se a necessidade de melhorias na implementação de ações preventivas com educação permanente e criação de novos protocolos por parte das instituições e organizações de saúde, bem como na adoção de medidas de biossegurança.
\end{abstract}

Palavras chave: atenção à saúde; acidentes de trabalho; Brasil.

\begin{abstract}
Objective: To characterize the quantitative evolution of accidents at work in human health care activities in the Northeast region.

Materials and Methods: This is a place-based eco-geographical study, through secondary data extracted from the database of the Ministry of Welfare and Social Assistance over the period 2007 to 2010.

Results: It was found that during the period studied, the state of Bahia presented the largest number of registered accidents at work. Typical accidents with Communication of a Work Accident (CWA) are configured as the main type in the ranking of the reasons/situation in which the consequences present the greater predominance of permanent disabilities.

Conclusions: Stands out the need for improvements in the implementation of preventive actions with permanent education and the creation of new protocols for the health institutions and organizations, as well as the adoption of biosecurity measures.
\end{abstract}

Keywords: health care; occupational accidents; Brazil.

\footnotetext{
${ }^{1}$ Enfermeira. Especialista em Enfermagem do Trabalho (Faculdade de Tecnologia e Ciências de Salvador - FTC/SSA) e Especialista em Urgência e Emergência (Universidade Castelo Branco-RJ). Docente da FTC/SSA.

${ }^{2}$ Fisioterapeuta. Mestrando em Saúde Coletiva (ISC/UFBA) e Especialista em Saúde Pública (Universidade Estácio de Sá-RJ). Docente do Departamento de Biofunção (ICS/UFBA) e da FTC/SSA.
} 


\section{INTRODUÇÃO}

O trabalho em saúde tem por característica, a integração entre aspectos intelectual e manual, onde os profissionais vinculam suas técnicas com execução de atividades manuais, e tratando-se do trabalhador da saúde, o seu propósito ou objetivo principal é a produção do cuidado ${ }^{1}$.

Os trabalhadores de saúde são todos os que se inserem direta ou indiretamente na atenção à saúde, em estabelecimento de saúde ou em tais atividades, podendo possuir ou não, formação especifica para o desempenho de funções inerentes ao setor ${ }^{2}$.

As atividades de atenção à saúde humana cobrem todas as formas de serviços relacionados à saúde, sejam aqueles prestados em hospitais, ambulatórios, consultórios, clínicas, centros de assistência psicossocial, unidades móveis de atendimento a urgências e remoções; sejam aqueles prestados nos domicílios ou atividades de apoio à gestão dos estabelecimentos de saúde ${ }^{3}$.

$\mathrm{Na}$ prática do cuidado, os trabalhadores da saúde estão expostos a riscos advindos do desenvolvimento de funções assistenciais diretas e indiretas, cuidados prestados diretamente a pacientes e à organização, bem como riscos relativos à limpeza e desinfecção de materiais, de equipamentos e do ambiente ${ }^{1}$.

Segundo alguns estudos, os acidentes de trabalho têm apresentado aumento significativo em diversos campos de ocupação, inclusive nas atividades da atenção à saúde humana, devido a exposições a riscos cada vez mais frequentes, com predomínio para os materiais biológicos ${ }^{4,5,6}$.

Os acidentes de trabalho são entendidos com aqueles ocorridos a serviço da empresa, provocando lesão corporal ou perturbação funcional, que cause a morte, perda, ou redução, permanente ou temporária, da capacidade para trabalho ${ }^{7}$.

O Ministério da Previdência e Assistência Social (MPAS) $^{7}$ classifica os acidentes de trabalho em notificados - correspondente àqueles em que a Comunicação de Acidentes de Trabalho (CAT) foi emitida e cadastrada junto ao Instituto Nacional do Seguro Social (INSS); acidentes sem CAT correspondentes àqueles cuja CAT não foi cadastrada no INSS; acidentes típicos - aqueles decorrentes da característica da atividade profissional desempenhada pelo acidentado; e os acidentes de trajeto - aqueles ocorridos no trajeto entre a residência e o local de trabalho do segurado e vice-versa.

No que tange às principais consequências, acidentes de trabalho podem resultar em incapacidades temporárias, caracterizadas pela limitação funcional para realização das atividades laborativas, por um período de 15 (quinze) dias consecutivos após afastamento da atividade; em incapacidades permanentes, relativas àquelas em que os segurados ficam permanentemente incapacitados para o exercício da sua atividade laboral; ou mesmo provocar a ocorrência de óbitos ${ }^{7}$.

Dentre os ambientes mais propícios para ocorrência de acidentes nas atividades de atenção à saúde, autores tem destacado o hospitalar, considerado insalubre, por agrupar indivíduos portadores de diversas enfermidades infectocontagiosas, por viabilizar diversos procedimentos com fluidos corpóreos, e por envolver a manipulação de artigos, roupas, lixos, materiais perfuro cortantes, dentre outros ${ }^{1,4,8}$.

Um estudo sobre acidentes de trabalho com trabalhadores de saúde identificou que a maior parte desses acidentes, incide, frequentemente, sobre profissionais de enfermagem, a exemplo dos auxiliares e técnicos, com predomínio de acometimento por material biológico. Também foram encontradas outras categorias profissionais vítimas desse agravo tais como, enfermeiros, médicos e profissionais da limpeza ${ }^{5}$.

Em virtude disso, tem aumentado o interesse das organizações de saúde e setores públicos, no que tange aos riscos ocupacionais a que estão sujeitos os trabalhadores da saúde, tendo em vista que tal situação afeta o desempenho de suas funções, podendo provocar sérios agravos à saúde, com possibilidades de perda da capacidade para o trabalho ${ }^{4}$.

Dessa maneira, estas questões têm se tornado objeto de intervenção e controle nacional e intersetorial, na medida em que suas repercussões impactam, não só sobre a saúde do trabalhador, mas também, sobre as organizações, estado e sociedade civil. Considerando esse cenário, as atividades de atenção à saúde humana 
se configuram como um contexto complexo, diante da diversidade de riscos e exposições que exigem controle permanente, para prevenir a ocorrência de acidentes de trabalho. Nessa perspectiva, a caracterização dos acidentes de trabalho torna-se uma possibilidade de compreensão de aspectos que possam auxiliar a tomada de decisão e repensar ações, bem como regulamentações em prol da vigilância e controle de tais agravos.

No entanto, apesar do aumento nos índices de acidentes de trabalho em atividades de atenção à saúde humana conforme a literatura revisada, na região Nordeste, há uma importante escassez de publicações científicas que caracterizem sua evolução quantitativa e debatam este problema. Desse modo, justifica-se a relevância deste estudo e de outros, que possam contribuir com informações que subsidiem a tomada de decisão, pelos órgãos e setores implicados na gestão dos riscos ocupacionais, no âmbito da atenção à saúde. Logo, este trabalho tem como objetivo caracterizar a evolução quantitativa dos acidentes de trabalho em atividades de atenção à saúde humana na região Nordeste.

\section{MATERIAIS E MÉTODOS}

Trata-se de um estudo ecológico de base territorial geográfica, com abordagem descritiva e comparativa, através de dados secundários que foram extraídos da base de dados do Ministério da Previdência e Assistência Social (MPAS). Segundo Rouquayrol e Filho $^{9}$, os estudos ecológicos abordam áreas geográficas bem delimitadas, analisando comparativamente variáveis globais, para produzir agregados que, por sua vez, servem de referência na construção dados. Quanto aos agregados, os estudos ecológicos podem ser caracterizados em dois tipos, a saber: de base territorial e agregados institucionais. Para este trabalho foi adotada a conformação de agregados territoriais, que utilizam a referência geográfica para a definição das suas unidades de informação ${ }^{9}$.

Para a coleta de dados foi consultada a base de dados INFOLOGO, na qual, encontra-se o Anuário Estatístico de Acidentes de Trabalho (AEAT), onde estão disponíveis dados sobre esses agravos, segundo diferentes categorias e ramos de atividade econômica ${ }^{10}$. Durante 0 acesso à base foram levantados apenas dados de acidentes referentes às atividades de atenção à saúde humana, objeto desse estudo. A sistematização dos dados ocorreu entre julho e agosto de 2012, sendo incluídas informações relativas aos anos de 2007 a 2010. Esse período foi escolhido tendo em vista a última atualização da Classificação Nacional das Atividades Econômicas (CNAE), já que os anos anteriores estavam agrupados segundo versões desatualizadas. Os anos posteriores a 2010 não foram incluídos nesse estudo, devido à indisponibilidade de dados organizados na base, durante o período de coleta.

As dimensões de análise foram definidas por conveniência, na medida em que foram consideradas aquelas disponíveis na base de dados. Portanto, neste trabalho serão descritas informações sobre a evolução quantitativa, considerando a existência ou ausência de Comunicação de Acidente de Trabalho (CAT); quantitativo de acidentes de trabalho por Unidades da Federação da região Nordeste; sobre acidentes de trabalho segundo motivo/situação desencadeante e suas principais consequências.

Foi realizada uma análise descritiva e comparativa, referente aos acidentes de trabalho, por meio da utilização do programa estatístico Microsoft Excel, versão 2010, sendo os resultados apresentados por frequências absoluta e relativa. Considerando que este trabalho não envolve a participação direta ou indireta de seres humanos, não houve necessidade de submetê-lo à apreciação por um comitê de ética, conforme especificado nas diretrizes da resolução 196/2012 do Conselho Nacional de Ética em Pesquisa (CONEP).

\section{RESULTADOS}

O comparativo dos acidentes de trabalho nas atividades de atenção à saúde humana entre a região Nordeste e o Brasil estão apresentado na figura 1. Em 2007 a porcentagem dos acidentes na região representou $8,2 \%$ do total de acidentes registrados no Brasil. Entre 2008 e 2009 houve uma diferença de apenas $0,4 \%$ na frequência de acidentes registrados, crescendo de $8,5 \%$ para $8,9 \%$. No ano de 2010 , 


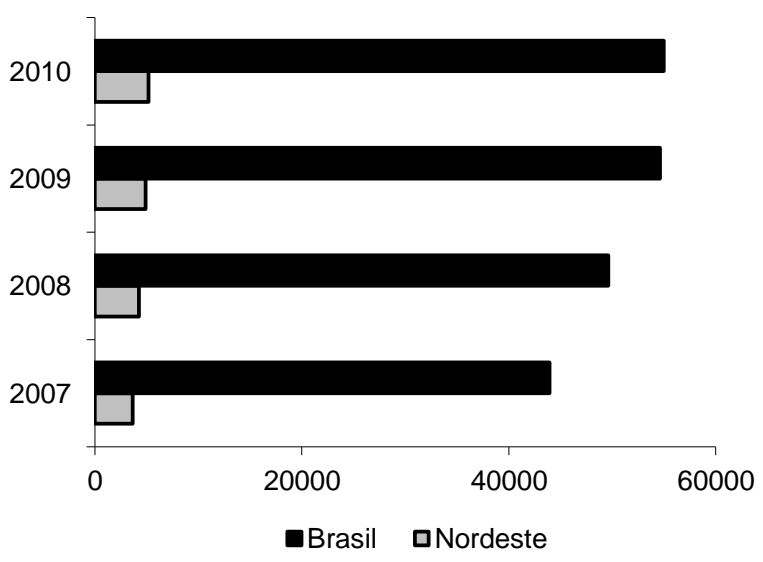

FIGURA 1 - Evolução comparativa dos acidentes de trabalho nas atividades de atenção à saúde humana.

percebe-se que a região Nordeste apresentou incremento da ordem de $9,4 \%$ em relação ao Brasil. Isto demonstra que de uma maneira geral, esta região, no espaço temporal analisado, apresentou aumento da frequência de acidentes de trabalho, contribuindo com parcelas importantes do total registrado no país.

A tabela 1 apresenta um panorama quantitativo dos acidentes de trabalho por Unidades da Federação (UF) na região. Observa-se que a região Nordeste apresentou 3.642 acidentes registrados em 2007. A frequência desse agravo alcançou 5.193 ocorrências em 2010, representando assim, um incremento de 29,8\% em relação à 2007.

Dentre os estados do Nordeste, destacou-se a Bahia, por apresentar a maior frequência de acidentes registrados na sequência temporal analisada. Entretanto, apesar disso, os dados demonstraram uma discreta redução de 29,9\% em 2007 para 27,2\% em 2010. Paralelamente à Bahia, o estado de Pernambuco se apresentou como o segundo com a maior frequência de acidentes de trabalho registrados, sobretudo a partir de 2008. Porém, os dados mostraram uma redução incipiente de $26,2 \%$ em 2007 para $25,8 \%$ em 2010 . Os estados da Paraíba e Piauí apresentaram o menor quantitativo de acidentes de trabalho em relação aos demais estados da região.

Conforme a figura 2, com relação à dimensão motivo/situação, os acidentes típicos com CAT apresentaram maior frequência quando comparado com os demais. Entretanto, observa-se que houve uma redução discreta de $0,7 \%$ entre o período de 2007 e

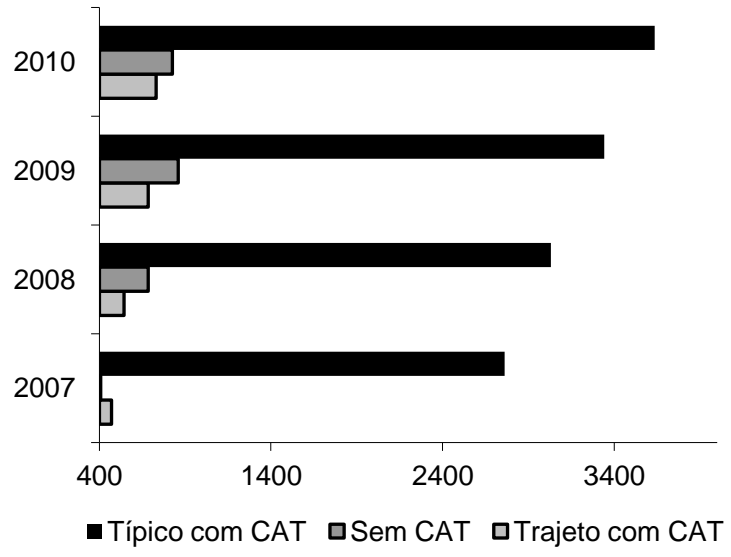

FIGURA 2 - Evolução quantitativa dos acidentes de trabalho nas atividades de atenção à saúde humana, segundo motivo ou situação. CAT: Comunicação de Acidente de Trabalho.

2008. Tendo em consideração os acidentes de trajeto com CAT, observa-se uma discreta redução de 2007 para 2008 de ordem de 0,2\%, porém apresentando crescimento em torno de 1,3\% em 2009 e mantendo-se em equilíbrio no ano de 2010. Com relação aos acidentes sem CAT, entre 2007 e 2008 houve um crescimento de $5 \%$. Por sua vez, o percentual de acidentes não registrados aumentou discretamente em $2009(17,5 \%)$, o que representou um crescimento de $1,4 \%$ com relação aos anos anteriores. A quantidade de acidentes de trabalho sem CAT, foi maior quando comparados com os acidentes de trajeto com CAT, denotando a ocorrência frequente de subnotificações (figura 2).

As principais consequências ocasionadas pelos acidentes de trabalho são apresentadas na tabela 2. É possível identificar que as incapacidades temporárias se configuram como a principal consequência, seguidas respectivamente da assistência médica, incapacidade permanente e óbitos. Em relação aos óbitos foi registrado crescimento contínuo de 2007 a 2010, com incremento de $0,03 \%$ ao final do período analisado. Referente à assistência médica, entre os anos de 2007 a 2010 houve um decréscimo, assumindo um percentual de $41,8 \%$. Quanto à incapacidade permanente, observou-se que os anos de 2007 e 2008 se mantiveram com $0,4 \%$, aumentando para $0,7 \%$ em 2010. No que concerne às incapacidades temporárias, os resultados demonstraram crescimento de 6,45\% entre 2007 e 2008, já que houve uma alteração no percentual de casos registrados de $53,6 \%$ para $60 \%$, 
TABELA 1 - Evolução quantitativa dos acidentes de trabalho nas atividades de atenção à saúde humana.

\begin{tabular}{|c|c|c|c|c|}
\hline \multirow{2}{*}{ Estado } & \multicolumn{4}{|c|}{ Quantitativo de acidentes $-\mathrm{n}^{\star}(\%)$} \\
\hline & 2007 & 2008 & 2009 & 2010 \\
\hline Alagoas & $396(10,8)$ & $527(12,3)$ & $523(10,7)$ & $515(9,9)$ \\
\hline Bahia & $1089(29,9)$ & $1164(27,3)$ & $1396(28,5)$ & $1416(27,2)$ \\
\hline Ceará & $445(12,2)$ & $545(12,7)$ & $731(14,9)$ & $713(13,7)$ \\
\hline Maranhão & $129(3,5)$ & $98(2,3)$ & $158(3,2)$ & $165(3,1)$ \\
\hline Paraíba & $53(1,4)$ & $78(1,8)$ & $81(1,6)$ & $88(1,6)$ \\
\hline Pernambuco & $957(26,2)$ & $1037(24,3)$ & $1164(23,8)$ & $1343(25,8)$ \\
\hline Piauí & $42(1,1)$ & $111(2,6)$ & $93(1,9)$ & $85(1,6)$ \\
\hline Rio Grande do Norte & $343(9,4)$ & $500(11,7)$ & $520(10,6)$ & $544(10,4)$ \\
\hline Sergipe & $188(5,1)$ & $200(4,6)$ & $221(4,5)$ & $324(6,2)$ \\
\hline Total & $3642(100)$ & $4260(100)$ & $4887(100)$ & $5193(100)$ \\
\hline
\end{tabular}

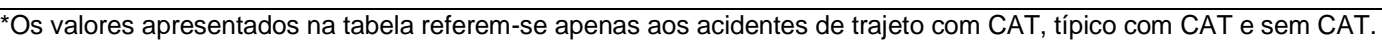

respectivamente neste período.

\section{DISCUSSÃO}

De acordo com um estudo realizado por Bakke e Araújo $^{6}$ em um hospital da Paraíba, entre os anos de 2001 a 2008, dos 38 acidentes de trabalho registrados, 34 corresponderam às atividades com profissionais de saúde. Canini et al. ${ }^{11}$, em 1998, identificaram 398 acidentes de trabalho com profissionais de saúde. Ruiz et al. $^{12}$, entre os anos de 2000 e 2001, também identificaram 861 ocorrências com este ramo de atividade profissional.

Estudos realizados em hospitais universitários no Rio de Janeiro e São Paulo demonstraram que ocorreram registros de, respectivamente, 265 e 53 acidentes de trabalho entre profissionais de saúde, com predomínio para exposição a líquidos corporais humanos ${ }^{13,14}$. Esses dados servem para ilustrar a magnitude que esse problema têm assumido no contexto das organizações de saúde.

Considerando esta realidade, mesmo passado mais de duas décadas do reconhecimento das ações de saúde do trabalhador como competência de atuação do sistema público de saúde, sob distintas estratégias e formas de organização institucional, torna-se irrefutável o desafio do SUS e das organizações, no controle dos acidentes de trabalho, sobretudo, entre os profissionais de saúde, seja pela complexidade do processo de trabalho, seja pela importância desses trabalhadores para a população.

Nessa perspectiva, com a implantação da Rede Nacional de Atenção Integral à Saúde do Trabalhador (RENAST) e seus respectivos Centros de Referência (CEREST), cria-se a expectativa de melhorias em questões relacionadas a notificações, investigações e respostas mais eficazes sobre os acidentes de trabalho. Porém, permanecem diversas dificuldades na implementação de estratégias de prevenção e controle para tais agravos, no âmbito do processo de trabalho em saúde, o que justifica novos estudos que contribuam para o planejamento e gestão de ações preventivas ${ }^{15}$.

Apesar do estado da Bahia se apresentar com o maior número de acidentes registrados na região, o pioneirismo deste estado deflagrou a criação da Política Estadual de Saúde do Trabalhador da Bahia (PEST), concomitantemente com o processo sociopolítico de institucionalização das ações de saúde do trabalhador, propostas na Constituição Federal (CF) de 1988. 
Embora tal fato pudesse justificar um maior grau de desenvolvimento da política de saúde do trabalhador, que impactasse positivamente sobre os indicadores de acidentes de trabalho no estado, no âmbito das atividades de atenção à saúde humana, percebe-se que há necessidade de maior e melhor controle, tendo em vista os resultados apresentados ${ }^{15,16}$.

De acordo com alguns autores, os trabalhadores da área da saúde estão expostos aos mesmos riscos (químicos, físicos e ergonômicos), a que se submetem os demais trabalhadores brasileiros. Porém, seus riscos de exposição se somam àqueles provenientes dos agentes biológicos, uma vez que em seu cotidiano, estes profissionais estão expostos ao contato com sangue e outros fluidos orgânicos, contaminados por uma grande variedade de micro-organismos desencadeadores de doenças ocupacionais, cuja causa mais comum de acidentes, correspondem às exposições a materiais perfurucortante $e^{17,18}$.

Silva et al. $^{18}$ afirmam que profissionais da área cirúrgica, enfermeiros, paramédicos, odontólogos e profissionais que atuam em setores de urgência e emergência, são considerados profissionais altamente predispostos à exposição ocupacional por fluidos e sangue. Apesar de tais informações, torna-se relevante destacar, que na base de dados consultada não foram encontrados disponíveis, dados sobre os acidentes, segundo categorias profissionais e tipo/fator desencadeante, o que de alguma forma comprometeu maior disponibilidade de informações, que pudesse enriquecer a caracterização regional desses acidentes.

Alguns estudos demonstram que dentre os profissionais da saúde, a equipe de enfermagem é sempre mais acometida por acidentes de trabalho, com frequência entre 30 a $50 \%$, devido à diversidade de fatores aos quais estão suscetíveis, dependendo da atividade realizada ${ }^{1,6}$. Paralelo a isso, pode-se incluir como fatores desencadeantes de acidentes de trabalho com profissionais da atenção à saúde, a situação ocupacional insatisfatória; o stress; a exposição a situações de elevada tensão emocional, associadas a longas jornadas de trabalho; condições de insalubridade do ambiente laboral, fadiga, baixos salários, dentre outros $^{1,6}$.
Souza et al. ${ }^{4}$ evidenciaram que dentre as categorias profissionais analisadas, "Estagiários Técnicos de Enfermagem", "Médicos Residentes" e "Acadêmicos de Medicina", também estão envolvidas em acidentes com materiais biológicos, totalizando $87,36 \%$ dos 166 acidentes de trabalho identificados no período estudado. Nesse estudo foram encontrados diferentes motivos/situações que corroboraram para ocorrência de acidentes de trabalho na região, dentre estes, os acidentes típicos ainda se configuram como os principais, embora sejam os mais sensíveis às ações de prevenção e controle das políticas públicas e das organizações, embora também sejam foco de Normas Regulamentadoras (NR) do Ministério do Trabalho e Emprego (MTE), a exemplo da NR $32^{19}$.

Corroborando com o descrito acima, Bakke e Araújo ${ }^{6}$ encontraram situações em que, frequentemente, trabalhadores da saúde re-encapeam ou desconectam manualmente, agulhas após uso no paciente, procedimento que é proibido pela Norma Regulamentadora (NR) $32^{19}$. Por outro lado, foi observado um quantitativo considerável de acidentes subnotificados. A CAT é o documento de notificação de acidentes de trabalho e doenças ocupacionais. Deve ser preenchido para garantir os direitos dos trabalhadores, na medida em que permite reconhecer legalmente, tanto a ocorrência do acidente, como a doença profissional adquirida. Este documento foi previsto oficialmente pela Lei 5.316/67 e deve ser emitido pelo setor de pessoal da empresa ou empregador e entregue ao posto do seguro social até o $1^{\circ}$ (primeiro) dia útil, após a ocorrência do acidente $^{19,20}$. Os dados desse estudo ratificaram a importância do problema referente às subnotificações na região, apesar de inúmeros esforços no que tange à informatização das notificações em todo o país. Isto dificulta a organização de dados essenciais para a compreensão do perfil epidemiológico desses acidentes, que possam orientar a tomada de decisão pelos setores e órgãos vigilância e controle ${ }^{21}$.

Em estudo sobre a invisibilidade dos acidentes de trabalho no município de Botucatu (SP), no ano de 1997, foi constatado que dos 37 acidentes identificados pelas entrevistas realizadas e enquadrados na emissão 
TABELA 2 - Evolução quantitativa dos acidentes de trabalho nas atividades de atenção à saúde humana, segundo consequência.

\begin{tabular}{lcccc}
\hline Consequência & $\begin{array}{c}\mathbf{2 0 0 7} \\
\mathbf{n}(\%)\end{array}$ & $\begin{array}{c}\mathbf{2 0 0 8} \\
\mathbf{n}(\%)\end{array}$ & $\begin{array}{c}\mathbf{2 0 0 9} \\
\mathbf{n}(\%)\end{array}$ & $\begin{array}{c}\mathbf{2 0 1 0} \\
\mathbf{n}(\%)\end{array}$ \\
\hline Óbitos & $1(0,02)$ & $3(0,06)$ & $4(0,07)$ & $2(0,03)$ \\
Incapacidade Permanente & $18(0,47)$ & $20(0,45)$ & $32(0,63)$ & $38(0,7)$ \\
Assistência Médica & $1734(45,8)$ & $1740(39,4)$ & $2020(40,0)$ & $2234(41,8)$ \\
Incapacidade Temporária & $2026(53,6)$ & $2653(60,0)$ & $2987(59,2)$ & $3061(57,3)$ \\
Total & $\mathbf{3 7 7 9 ( 1 0 0 )}$ & $\mathbf{4 4 1 6 ( 1 0 0 )}$ & $\mathbf{5 0 4 3 ( 1 0 0 )}$ & $\mathbf{5 3 3 5 ( 1 0 0 )}$ \\
\hline
\end{tabular}

de CAT, $54 \%$ não haviam sido registrados no INSS local. Esses resultados evidenciam que os registros da Previdência Social (PS) captaram apenas 22,4\% dos acidentes locais ${ }^{21}$.

Diante dessa realidade, o problema de subnotificação reforça a necessidade de campanhas educativas e preventivas por parte do estado, empresas e instituições. São necessárias também, fiscalizações por parte do Ministério do Trabalho e Emprego (MTE) e do Ministério da Previdência e Assistência Social (MPAS). Isso reforça a importância do registro dos trabalhadores, bem como maiores penalizações das organizações que descumpram a legislação, a fim de garantir melhores condições de trabalho ${ }^{19,20,22}$.

Cabe refletir ainda, sobre a incidência de incapacidades temporárias ou permanentes, que ocasionam impactos financeiros, tanto para o governo, no que diz respeito aos benefícios a serem pagos aos segurados, quanto para as organizações, pois levam à instabilidade e perdas de produtividade, pelo número reduzido de trabalhadores, acarretando em reposição de mão-de-obra, além de multas e substituições ${ }^{22}$. Para trabalhadores, tal situação implica em diminuição ou falta de empregabilidade, devido à lesão, perda da qualidade de vida e bem-estar, além de redução da motivação, pois reflete diretamente sobre a aptidão do trabalhador no que tange ao retorno para o trabalho, aumentando absenteísmos, perda de imagem ou autoconfiança, como por exemplo, nas lesões que corroboram para mutilações de membros ${ }^{22}$.

Este estudo teve como principais limitações metodológicas, a indisponibilidade de dados mais atuais na base, referentes aos anos posteriores a 2010, durante o período da sistematização; a inexistência de dados sobre acidentes de trabalho no ramo de atividade econômica analisado segundo sexo, grau de escolaridade, faixa etária, cor e categorias profissionais da saúde. Há de se considerar ainda, a limitação decorrente da escassez de publicação sobre acidentes de trabalho nas atividades de atenção à saúde humana, bem como ausência de estudos que pudessem fornecer dados sobre esse agravo entre os estados do Nordeste, que permitissem uma comparabilidade mais específica com os dados desse estudo.

Em síntese, percebeu-se que os dados deste estudo, demonstraram crescimento significativo dos acidentes de trabalho nas atividades de atenção humana na região Nordeste, com relação ao total de acidentes registrados, neste mesmo período, no Brasil. Observouse também, que dentre os estados analisados, a Bahia se destacou com o maior número de acidentes de trabalho da região. Quanto aos motivos/situações, os acidentes típicos com CAT lideraram o ranking, apresentando maior quantitativo quando comparado com acidentes de trajeto com CAT e sem CAT. Destacaram-se ainda, os acidentes sem CAT, por apresentar aumento considerável no período analisado. Com relação às consequências, as incapacidades temporárias destacaram-se como as principais.

Assim, considera-se importante que os profissionais de saúde devem atentar-se para o autocuidado, tomando precauções apropriadas, no âmbito da biossegurança, para a realização de suas atividades laborativas, prevenindo assim, a ocorrência de acidentes. Entretanto, cabe também às instituições e organizações, adotarem medidas adequadas como a criação de novos protocolos, que visem estratégias para redução dos riscos ambientais e, sobretudo, sejam mais 
proativas no processo de registro dos acidentes ocorridos, na adoção de medidas preventivas que promovam a educação em serviço, disponibilizando equipamentos de proteção individual aos seus colaboradores, bem como a supervisão adequada dos profissionais contratados e dos seus estagiários. Tornase imprescindível a realização de pesquisas empíricas que enfoquem os acidentes de trabalho nas atividades de atenção à saúde na região Nordeste, seja como fonte de análise diagnóstica mais aprofundada, seja como ferramenta de avaliação e reorientação das políticas públicas de atenção à saúde do trabalhador, tendo em vista a escassez de estudos empíricos na literatura, que dessem subsídio aos dados secundários extraídos neste trabalho.

\section{REFERÊNCIAS}

1. Pinho DLM, Rodrigues CM, Gomes GP. Perfil dos acidentes de trabalho no Hospital Universitário de Brasília. Rev Bras Enferm. 2007 Jun [acesso em 2012 ago19]; 60(3):291-4. Disponível em http://www.scielo.br/pdf/reben/ v60n3/a08.pdf. Acesso em: 20 jul. 2012.

2. Geovanella L. Políticas e sistema de saúde no Brasil. Rio de Janeiro: Fiocruz; 2008.

3. Brasil. Ministério do Planejamento, Orçamento e Gestão. Comissão de Classificação Nacional das Atividades Econômicas. Brasília: Ministério do Planejamento, Orçamento e Gestão; 2007. Disponível em: http://www.cnae.ibge.gov.br/estrutura.asp?TabelaBusca= CNAE_200@CNAE\%202.0@0@cnae@0. Acesso em: 15 jul. 2012.

4. Souza R T, BicaL CG, Mondadori CS, Ranzi AD. Avaliação de acidentes de trabalho com materiais biológicos em médicos residentes, acadêmicos e estagiários de um hospital-escola de Porto Alegre. Rev Bras Educ Med 2012 [acesso em 2012 jul 20] fev; 36(1):118-24. Disponível em http://www.scielo.br/pdf/rbem/ v36n1/a16v36n1.pdf.

5. Valim MD, Marziale MHP. Avaliação da exposição ocupacional a material biológico em serviços de saúde. Texto Contexto-Enferm. 2011[acesso em 2012 ago 2]; 2:138-46. Disponível em: http://www.scielo.br/pdf/tce/ v20nspe/v20nspea18.pdf

6. Bakke HA, Araújo NMC. Acidentes de trabalho com profissionais de saúde de um hospital universitário. Prod. 2010 [acesso em 2012 ago 2]; 20(4):669-76. Disponível em: http://www.scielo.br/pdf/prod/v20n4/aop00040109.pdf

7. Brasil. Ministério do Trabalho e Emprego. Lei 6367 de 19 de outubro de 1976. Dispõe sobre o seguro de acidentes do trabalho a cargo do INPS e dá outras providências. Diário Oficial [da] República Federativa do Brasil. 1976 out 21 [acesso em 2012 ago 2]. Disponível em: http://www010.dataprev.gov.br/sislex/paginas/42/1976/636 7.htm.

8. Machado MRM, Machado FA. Acidentes com material biológico em trabalhadores de enfermagem do Hospital Geral de Palmas (TO). Rev Bras Saúde Ocup. 2011 [acesso em 2012 ago 19]; 36(124):274-81. Disponível em: http://www.scielo.br/pdf/rbso/v36n124/a11v36n124.pdf

9. Rouquayrol $\mathrm{MZ}$, Filho $\mathrm{N}$ de $\mathrm{A}$. Introdução à Epidemiologia. São Paulo: Guanabara Koogan; 2002.

10. Brasil. Ministério da Previdência e Assistência Social. Anuário Estatístico da Previdência Social (2007-2010) [acesso em 2012 jul 20]. Disponível em http://www.mpas.gov.br/arquivos/office/3_091125-174455479.pdf

11. Canini SRMS, Gir E, Hayashida M, Machado AA. Acidentes perfurocortantes entre trabalhadores de enfermagem de um hospital universitário do interior paulista. Rev Latino-Am Enferm. 2002 [acesso em 2012 ago 19]; 10(2):172-8. Disponível em: http://www.scielo.br/ pdf/rlae/v10n2/10511.pdf.

12. Ruiz MT, Barboza DB, Soler ZASG. Acidentes de trabalho: um estudo sobre esta ocorrência em um hospital geral. Revista Arq de Ciênc da Saúde. 2004 [acesso em 201228 ago 28];11(4):219-24. Disponível em http://www.cienciasdasaude.famerp.br/ racs_ol/Vol-11 4/05\%20-\%20id\%2046.pdf.

13. Osório C, Machado JMH, Minayo-Gomez C. Proposição de um método de análise coletiva dos acidentes de trabalho no hospital. Cad Saúde Pública. 2005 [acesso em 2012 ago 19]; 21(2):517-24. Disponível em: http://www.scielo.br/pdf/csp/v21n2/18.pdf.

14. Balsamo AC, Felli VEA. Estudo sobre os acidentes de trabalho com exposição aos líquidos corporais humanos em trabalhadores da saúde de um hospital universitário. Rev Latino-Am Enferm. 2006 [acesso 2012 em jul 20]; 14(3):346-53. Disponível em http://www.scielo.br/pdf/rlae/ v14n3/v14n3a07.pdf.

15. Galdino A, Santana VS, Ferrite S. Os Centros de Referência em Saúde do Trabalhador e a notificação de acidentes de trabalho no Brasil. Cad Saúde Pública. 2012 [acesso em 2012 jul 25]; 28(1):145-59. Disponível em http://www.scielo.br/pdf/csp/v28n1/15.pdf

16. Brasil. Ministério da Saúde. Centro de Referência em Saúde do Trabalhador. Brasília: Ministérios da Saúde; 2012 [acesso em 2012 set 2]. Disponível em: <http://www.cerest.rn.gov.br/contentproducao/aplicacao/se sap_cerest/cerest/gerados/saude_trabalhador.asp.

17. Almeida CAF, Benatti MCC. Exposições ocupacionais por fluidos corpóreos entre trabalhadores da saúde e sua adesão à quimioprofilaxia. Rev Esc Enferm. 2007 [acesso em 2012 set 2]; 41(1):120-6. Disponível em: http://www.scielo.br/pdf/reeusp/v41n1/v41n1a15.pdf

18. Silva JA, Paula VS, Almeida J de A. Investigação de acidentes biológicos entre profissionais de saúde. Esc. Anna Nery [online]. 2009 [acesso em 2012 ago 10]; 13(3):508-16. Disponível em http://www.scielo.br/pdf/ean/ v13n3/v13n3a08.pdf.

19. Brasil. Ministério do Trabalho e Emprego. Portaria 1.748 de 30 de agosto de 2011. Dispõe sobre a Norma Regulamentadora 32. Diário Oficial [da] República Federativa do Brasil. 2011 out 21 [acesso em 2012 ago 2]. Disponível em: <http://portal.mte.gov.br/data/files/ 8A7C812D36A280000138812EAFCE19E1/NR-32\%20 (atualizada\%202011).pdf.

20. Brasil. Ministério da Saúde. Política Nacional de Saúde do Trabalhador: Proposta para Consulta Pública. Secretaria de Atenção à Saúde. Departamento de Ações Programáticas Estratégicas. Brasília: Ministério da Saúde; 2004. p. 1-41.

21. Binder MCP, Cordeiro R. Sub-registro de acidentes de trabalho em localidade do Estado de São Paulo, 1997. Rev Saúde Pública 2003 [acesso em 2012 ago 15]; 37(4):409-16. Disponível em: http://www.scielo.br/pdf/rsp/ v37n4/16774.pdf. 
22. Brasil. Ministério da Saúde. Notificação de Acidentes do Trabalho Fatais, Graves e com Crianças e Adolescentes. Secretaria de Atenção à Saúde. Série A Normas e Manuais Técnicos. Brasília: Ministério da Saúde; 2006. [acesso em 2012 set 2]. 32 p. Disponível em: http://portal.saude.gov.br/portal/arquivos/pdf/trabalho_infa ntil_protocolos_web.pdf.

\section{Endereço para correspondência:}

Ítalo Ricardo Santos Aleluia

Al. Piatã, Cond. Vale das Flores, Ed. Tulipa $n^{\circ} 16$

Salvador/BA - CEP 40275-010

Telefone: + 557182066342

E-mail: italoaleluia@yahoo.com.br 